\title{
Optimal Application Timing of Pest Control Tactics in Nonautonomous Pest Growth Model
}

\author{
Shujuan Zhang, Juhua Liang, and Sanyi Tang \\ College of Mathematics and Information Science, Shaanxi Normal University, Xian 710062, China \\ Correspondence should be addressed to Juhua Liang; juhualiang81@gmail.com
}

Received 28 March 2014; Accepted 25 May 2014; Published 25 June 2014

Academic Editor: Shengqiang Liu

Copyright (C) 2014 Shujuan Zhang et al. This is an open access article distributed under the Creative Commons Attribution License, which permits unrestricted use, distribution, and reproduction in any medium, provided the original work is properly cited.

Considering the effects of the living environment on growth of populations, it is unrealistic to assume that the growth rates of predator and prey are all constants in the models with integrated pest management (IPM) strategies. Therefore, a nonautonomous predator-prey system with impulsive effect is developed and investigated in the present work. In order to determine the optimal application timing of IPM tactics, the threshold value which guarantees the stability of pest-free periodic solution has been obtained firstly. The analytical formula of optimal application timings within a given period for different cases has been obtained such that the threshold value is the smallest, which is the most effective in successful pest control. Moreover, extensively numerical investigations have also been confirmed our main results and the biological implications have been discussed in more detail. The main results can guide the farmer to design the optimal pest control strategies.

\section{Introduction}

Recently, many ecologists are becoming increasingly concerned with the questions of pest control and designing the optimal control strategies. It is well known that the pesticides are still the main tactics for controlling pests, because the pesticides are relatively cheap and can be easily applied. But spraying insecticides for a long time may trigger " $3 \mathrm{R}$ " questions (resistance, resurgence, and residue); then, the Department of Agricultural Ecology proposed the integrated pest management (IPM) strategies $[1,2]$.

IPM is a long term management strategy, which includes chemical (insecticides), biological (releasing the natural enemy), agricultural control (crop rotation), and physics methods (utilizing light to trap and kill pest) $[3,4]$. IPM which has been proved by experiment is more effective than any single control strategy. But when should we release the natural enemy and what proportion do we need to kill the pest by spraying pesticide? Undoubtedly, the mathematical models can help us to design the optimal control tactics and, in particular, help us to predict the density of pest population and to determine optimal application timing of IPM strategies (see [5-8]).

Volterra first proposed a simple predator-prey system, which has been extended and modified in many ways $[9,10]$.
In recent years, continuous or discrete predator-prey systems concerning IPM strategies have been developed and investigated intensively in [11-13]. Considering the interventions by human such as a periodic spraying pesticide and a constant periodic releasing for the predator, the impulsive differential equations with fixed moments were employed to model the interventions, and consequently the Lotka-Volterra system has been extended [14]. However, one of the major assumptions in those publications was that all the growth rates of predator and prey are constants. However, many ecologists have shown that the growth of populations of various species is affected by the special living environment including the seasons, weather conditions, and food supply [15]. Therefore, it is more realistic to consider the effects of periodic parameters on the dynamics of predator-prey models. Therefore, nonautonomous predator-prey systems with impulsive effect have been developed and investigated in $[10,16,17]$.

However, those works mainly focused on the effects of periodic perturbations on the dynamics; the interesting questions concerning the effects of successful pest control, in particular how to apply the IPM tactics in periodic environment and to determine the optimal application timing, have not been addressed in more detail. 
Thus, in the present work, a nonautonomous predatorprey model with periodic perturbations and periodic impulsive effects is developed and investigated. Firstly, the stability of pest-free periodic solution and its threshold condition have been investigated, which can be used for determining the optimal timing of IPM applications. Secondly, we assume that only one-impulsive controlling has been applied within a given period, and the optimal application timing has been determined and consequently the analytical formula is also provided, under which the threshold value reaches its minimum. Then, we assume that two-impulsive controlling or more impulsive controlling has been applied within a given period, and similarly the optimal time points and their analytical formula have also been obtained and provided. Moreover, the biological implications have been discussed in more detail. Finally, some numerical simulations concerning the main results have been done. We conclude that the main results can help us to design the optimal pest control strategies.

\section{The Periodic Integrated Pest Control Strategies}

2.1. Autonomous ODE Model with Multi-Impulsive Effects. Assume that the pest population follows the classical logistic growth system and that pest control by spraying pesticides and releasing natural enemies is implemented at some fixed times for each crop season. Denote the size of the pest and the natural enemy populations at time $t$ by $x(t)$ and $y(t)$, respectively. Assume that pests are killed by pesticides at a proportional rate $p_{k}\left(0 \leq p_{k}<1\right)$ and the natural enemy is released by a constant $\sigma$ at time $\tau_{k}$. Therefore, we have the following system with impulsive effects at fixed moments:

$$
\begin{aligned}
& \frac{d x(t)}{d t}=r x(t)[1-a x(t)]-b x(t) y(t), \\
& \frac{d y(t)}{d t}=y(t)[c x(t)-\delta], \\
& \quad t \neq \tau_{k}, \quad(k=1,2, \ldots), x\left(\tau_{k}^{+}\right)=\left(1-p_{k}\right) x\left(\tau_{k}\right), \\
& y\left(\tau_{k}^{+}\right)=y\left(\tau_{k}\right)+\sigma, \\
& t=\tau_{k},
\end{aligned}
$$

where $r$ is the intrinsic growth rate of pest population, $a$ denotes the carrying capacity parameter, $b$ is the attack rate of predator, $c$ represents conversion efficiency, and $\delta$ is the death rate of predator. System (1) is said to be a $T$ periodic system if there exists a positive integer $q$ such that $p_{k+q}=p_{k}$, $\tau_{k+q}=\tau_{k}+T$. This implies that, in each period $T, q$ times of the pesticide applications are used and $q$ times of the natural enemy releases are applied.

The dynamical behavior and biological implications of system (1) have been extensively studied in [18]. It follows from the literature [18] that if $\exp (-\delta T)<1$, then system (1) has a "pest-eradication" periodic solution $\left(0, y^{T}(t)\right)$ over the $n$th time interval $n T<t \leq(n+1) T$ with

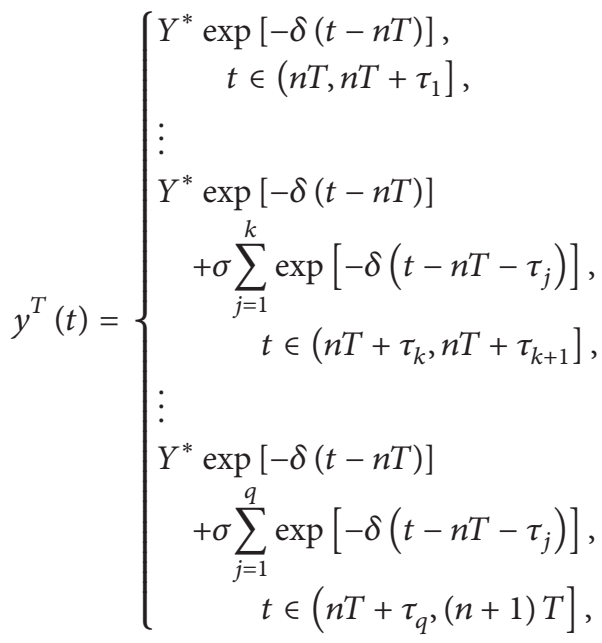

where $Y^{*}=\left(\sigma \sum_{j=1}^{q} \exp \left(-\delta\left(T-\tau_{j}\right)\right)\right) /(1-\exp (-\delta T))$ and $\left(0, y^{T}(t)\right)$ is globally asymptotically stable provided that $R_{0}<$ 1 with

$$
R_{0} \triangleq \prod_{i=1}^{q}\left(1-p_{i}\right) \exp \left(r T-\frac{b q \sigma}{\delta}\right)
$$

The analytical formula defined above clearly shows how the key parameters affect the threshold value $R_{0}$, which can be used to design the optimal control strategies such that the threshold value $R_{0}$ is the smallest. We will address those points in the following for more generalized model.

2.2. Nonautonomous ODE Model with Multi-Impulsive Effects. However, it is well known that the growth of populations of various species is affected by several factors such as the seasons, weather conditions, and food supply, which can be described by using the periodic coefficients in model (1); that is, we have the following model:

$$
\begin{aligned}
& \frac{d x(t)}{d t}=r(t) x(t)[1-a(t) x(t)]-b(t) x(t) y(t), \\
& \frac{d y(t)}{d t}=y(t)[c(t) x(t)-\delta(t)],
\end{aligned}
$$$$
t \neq \tau_{k}
$$

$$
\begin{array}{r}
x\left(\tau_{k}^{+}\right)=\left(1-p_{k}\right) x\left(\tau_{k}\right), \\
y\left(\tau_{k}^{+}\right)=y\left(\tau_{k}\right)+\sigma_{k}, \\
t=\tau_{k},
\end{array}
$$

where $r(t), a(t), b(t), c(t)$, and $\delta(t)$ are continuous $T$ periodic functions. System (4) is said to be a $T$ periodic system if there exists a positive integer $q$ such that $p_{k+q}=p_{k}, \sigma_{k+q}=\sigma_{k}$, and $\tau_{k+q}=\tau_{k}+T$. 
In order to analyze the dynamics of the pest population in system (4), the following subsystem is useful:

$$
\begin{array}{ll}
\frac{d y(t)}{d t}=-\delta(t) y(t), & t \neq \tau_{k}, \\
y\left(\tau_{k}^{+}\right)=y\left(\tau_{k}\right)+\sigma_{k}, & t=\tau_{k}
\end{array}
$$

and we have the following main results for subsystem (5).

Lemma 1. The subsystem (5) has a positive periodic solution $y^{T}(t)$ and for every solution $y(t)$ of (5) one has $\left|y(t)-y^{T}(t)\right| \rightarrow$ 0 as $n \rightarrow \infty$, where

$$
\begin{aligned}
& y^{T}(t)=\left\{\begin{array}{c}
Y_{n}^{*} \exp \left[-\int_{n T}^{t} \delta(s) d s\right], \\
t \in\left(n T, n T+\tau_{1}\right], \\
\vdots \quad \sum_{j=1}^{k} \sigma_{j} \exp \left[-\int_{n T+\tau_{j}}^{t} \delta(s) d s\right], \\
\quad t \in\left(n T+\tau_{k}, n T+\tau_{k+1}\right], \\
\vdots \quad \exp \left[-\int_{n T}^{t} \delta(s) d s\right] \\
Y_{n}^{*} \exp \left[-\int_{n T}^{t} \delta(s) d s\right] \\
+\sum_{j=1}^{q} \sigma_{j} \exp \left[-\int_{n T+\tau_{j}}^{t} \delta(s) d s\right], \\
t \in\left(n T+\tau_{q},(n+1) T\right],
\end{array}\right. \\
& Y_{n}^{*}=\frac{\sum_{j=1}^{q} \sigma_{j} \exp \left[-\int_{n T+\tau_{j}}^{(n+1) T} \delta(s) d s\right]}{1-\exp \left[-\int_{n T}^{(n+1) T} \delta(s) d s\right]},
\end{aligned}
$$

$n \in \mathcal{N}$ and $\mathcal{N}=\{0,1,2, \ldots\}$.

Proof. In any given time interval $(n T,(n+1) T]$ (where $n$ is a natural number), we investigate the dynamical behavior of system (5). In fact, integrating the first equation of system (5) from $n T$ to $n T+\tau_{1}$ yields

$$
y(t)=y(n T) \exp \left[-\int_{n T}^{t} \delta(s) d s\right], \quad t \in\left(n T, n T+\tau_{1}\right] .
$$

At time $n T+\tau_{1}$, the $\sigma_{1}$ natural enemy is released; thus, we have

$$
y\left(\left(n T+\tau_{1}\right)^{+}\right)=y(n T) \exp \left[-\int_{n T}^{n T+\tau_{1}} \delta(s) d s\right]+\sigma_{1} .
$$

Again, integrating the first equation of system (5) from $n T+\tau_{1}$ to $n T+\tau_{2}$, one yields

$$
\begin{array}{r}
y(t)=y\left(\left(n T+\tau_{1}\right)^{+}\right) \exp \left[-\int_{n T+\tau_{1}}^{t} \delta(s) d s\right], \\
t \in\left(n T+\tau_{1}, n T+\tau_{2}\right] .
\end{array}
$$

At time $n T+\tau_{2}$, the $\sigma_{2}$ natural enemy is released, and

$$
\begin{aligned}
y\left(\left(n T+\tau_{2}\right)^{+}\right)= & y(n T) \exp \left[-\int_{n T}^{n T+\tau_{2}} \delta(s) d s\right] \\
& +\sigma_{1} \exp \left[-\int_{n T+\tau_{1}}^{n T+\tau_{2}} \delta(s) d s\right]+\sigma_{2} .
\end{aligned}
$$

By induction, we get

$$
\begin{aligned}
y(t)= & y(n T) \exp \left[-\int_{n T}^{t} \delta(s) d s\right] \\
& +\sum_{j=1}^{q} \sigma_{j} \exp \left[-\int_{n T+\tau_{j}}^{t} \delta(s) d s\right]
\end{aligned}
$$

for all $t \in\left(n T+\tau_{q},(n+1) T\right]$. Therefore, we have

$$
\begin{aligned}
y((n+1) T)= & y(n T) \exp \left[-\int_{n T}^{(n+1) T} \delta(s) d s\right] \\
& +\sum_{j=1}^{q} \sigma_{j} \exp \left[-\int_{n T+\tau_{j}}^{(n+1) T} \delta(s) d s\right] .
\end{aligned}
$$

Denote $Y_{n+1}=y((n+1) T)$; then, we have the following difference equation:

$$
\begin{aligned}
Y_{n+1}= & Y_{n} \exp \left[-\int_{n T}^{(n+1) T} \delta(s) d s\right] \\
& +\sum_{j=1}^{q} \sigma_{j} \exp \left[-\int_{n T+\tau_{j}}^{(n+1) T} \delta(s) d s\right],
\end{aligned}
$$

which has a unique steady state

$$
Y_{n}^{*}=\frac{\sum_{j=1}^{q} \sigma_{j} \exp \left[-\int_{n T+\tau_{j}}^{(n+1) T} \delta(s) d s\right]}{1-\exp \left[-\int_{n T}^{(n+1) T} \delta(s) d s\right]} .
$$

Thus, there is a periodic solution of system (5), denoted by $y^{T}(t)$, which is given in (6). For the stability of $y^{T}(t)$, it follows from (12) and the formula of $y^{T}(t)$ that

$$
\begin{aligned}
\left|y(t)-y^{T}(t)\right| & \mid y(n T) \exp \left[-\int_{n T}^{t} \delta(s) d s\right] \\
+ & \sum_{j=1}^{k} \sigma_{j} \exp \left[-\int_{n T+\tau_{j}}^{t} \delta(s) d s\right] \\
& -Y_{n}^{*} \exp \left[-\int_{n T}^{t} \delta(s) d s\right] \\
& -\sum_{j=1}^{k} \sigma_{j} \exp \left[-\int_{n T+\tau_{j}}^{t} \delta(s) d s\right] \mid
\end{aligned}
$$




$$
\begin{gathered}
=\left|y(n T)-Y_{n}^{*}\right| \exp \left[-\int_{n T}^{t} \delta(s) d s\right] \\
\longrightarrow 0 \quad \text { as } \quad n \longrightarrow \infty \\
\quad t \in\left(n T+\tau_{k}, n T+\tau_{k+1}\right]
\end{gathered}
$$

and then the results of Lemma 1 follow. This completes the proof of Lemma 1.

Based on the conclusion of Lemma 1, there exists a "pestfree" periodic solution of system (4) over the $n$th time interval $n T<t \leq(n+1) T$, and we have the following threshold conditions.

Theorem 2. Let

$$
\begin{aligned}
R_{0}= & \prod_{i=1}^{q}\left(1-p_{i}\right) \\
& \times \exp \left[\int_{n T}^{(n+1) T} r(t) d t\right. \\
& -\sum_{j=0}^{q-1} \int_{n T+\tau_{j}}^{n T+\tau_{j+1}} b(t) y^{T}(t) d t \\
& \left.-\int_{n T+\tau_{q}}^{(n+1) T} b(t) y^{T}(t) d t\right] ;
\end{aligned}
$$

then the pest-free periodic solution $\left(0, y^{T}(t)\right)$ of system (4) is globally asymptotically stable if $R_{0}<1$.

Proof. Firstly, we prove the local stability. Define $x(t)=u(t)$, $y(t)=y^{T}(t)+v(t)$; there may be written

$$
\left(\begin{array}{l}
u(t) \\
v(t)
\end{array}\right)=\Phi(t)\left(\begin{array}{l}
u(0) \\
v(0)
\end{array}\right)
$$

where $\Phi(t)$ satisfies

$$
\frac{d \Phi}{d t}=\left(\begin{array}{cc}
r(t)-b(t) y^{T}(t) & 0 \\
c(t) y^{T}(t) & -\delta(t)
\end{array}\right) \Phi(t),
$$

and $\Phi(0)=I$ denoted the identity matrix. The resetting of the third and fourth equations of (4) becomes

$$
\left(\begin{array}{l}
u\left(n T+\tau_{k}^{+}\right) \\
v\left(n T+\tau_{k}^{+}\right)
\end{array}\right)=\left(\begin{array}{cc}
1-p_{k} & 0 \\
0 & 1
\end{array}\right)\left(\begin{array}{c}
u\left(n T+\tau_{k}\right) \\
v\left(n T+\tau_{k}\right)
\end{array}\right)
$$

Hence, if both eigenvalues of

$$
M=\left(\begin{array}{ccc}
\prod_{i=1}^{q}\left(1-p_{i}\right) & 0 \\
& 0 & 1
\end{array}\right) \Phi(T)
$$

have absolute value less than one, then the periodic solution $\left(0, y^{T}(t)\right)$ is locally stable. In fact, the two Floquet multiplies are thus

$$
\begin{gathered}
\mu_{1}=\prod_{i=1}^{q}\left(1-p_{i}\right) \exp \left[\int_{n T}^{(n+1) T}\left(r(t)-b(t) y^{T}(t)\right) d t\right] \\
\mu_{2}=\exp \left[-\int_{n T}^{(n+1) T} \delta(t) d t\right]<1
\end{gathered}
$$

according to Floquet theory (see [19-21] and the references therein), the solution $\left(0, y^{T}(t)\right)$ is locally stable if $\left|\mu_{1}\right|<1$; that is, if (17) holds true, the solution of system (4) is locally stable.

In the following, we will prove the global attractivity of $\left(0, y^{T}(t)\right)$. It follows from the second equation of system (4) that we have $d y(t) / d t>-\delta(t) y(t)$, and consider the following impulsive differential equation:

$$
\begin{aligned}
& \frac{d u(t)}{d t}=-\delta(t) u(t), \quad t \neq \tau_{k}, \\
& u\left(\tau_{k}^{+}\right)=u\left(\tau_{k}\right)+\sigma_{k}, \quad t=\tau_{k},
\end{aligned}
$$

where $\delta(t)$ is continuous $T$ periodic function and $\sigma_{k+q}=\sigma_{k}$, $\tau_{k+q}=\tau_{k}+T$.

According to Lemma 1 and the comparison theorem on impulsive differential equations, we get $y(t) \geq u(t)$ and $u(t) \rightarrow y^{T}(t)$ as $t \rightarrow \infty$. Therefore,

$$
y(t) \geq u(t)>y^{T}(t)-\epsilon
$$

holds for $\epsilon(\epsilon>0)$ small enough and all $t$ large enough. Without loss of generality, we assume that (24) holds for all $t \geq 0$. Thus, we have

$$
\begin{gathered}
\frac{d x(t)}{d t} \leq r(t) x(t)-b(t) x(t)\left[y^{T}(t)-\epsilon\right], \quad t \neq \tau_{k}, \\
x\left(\tau_{k}^{+}\right)=\left(1-p_{k}\right) x\left(\tau_{k}\right), \quad t=\tau_{k} .
\end{gathered}
$$

Again, from the comparison theorem on impulsive differential equations, we get

$$
\begin{aligned}
& x\left(n T+\tau_{1}\right) \\
& \leq x(n T) \exp \left\{\int_{n T}^{n T+\tau_{1}}\left[r(t)-b(t)\left(y^{T}(t)-\epsilon\right)\right] d t\right\}, \\
& x\left(n T+\tau_{2}\right) \\
& \leq\left(1-p_{1}\right) x\left(n T+\tau_{1}\right) \\
& \quad \times \exp \left\{\int_{n T+\tau_{1}}^{n T+\tau_{2}}\left[r(t)-b(t)\left(y^{T}(t)-\epsilon\right)\right] d t\right\}
\end{aligned}
$$




$$
\begin{aligned}
\leq & \left(1-p_{1}\right) x(n T) \\
& \times \exp \left\{\sum_{j=0}^{1} \int_{n T+\tau_{j}}^{n T+\tau_{j+1}}\left[r(t)-b(t)\left(y^{T}(t)-\epsilon\right)\right] d t\right\}, \\
\vdots & x((n+1) T) \\
\leq & \prod_{i=1}^{q}\left(1-p_{i}\right) x(n T) \\
& \times \exp \left\{\sum_{j=0}^{q-1} \int_{n T+\tau_{j}}^{n T+\tau_{j+1}}\left[r(t)-b(t)\left(y^{T}(t)-\epsilon\right)\right] d t\right. \\
= & \prod_{i=1}^{q}\left(1-\int_{i}\right) x(n T) \\
& \times \exp \left\{\int_{n T}^{(n+1) T}[r(t)+b(t) \epsilon] d t\right. \\
\triangleq & \left.x(n T) R_{\epsilon}, \quad-\int_{n T+\tau_{q}}^{q-1} \int_{j=0}^{n T+\tau_{q}} \int_{n T+\tau_{j}}^{(n+1) T} b(t) y^{T}(t) d t\right\} \\
&
\end{aligned}
$$

where $\tau_{0}=0$ and

$$
\begin{aligned}
R_{\epsilon}=\prod_{i=1}^{q}\left(1-p_{i}\right) \exp \left\{\int_{n T}^{(n+1) T}[r(t)+b(t) \epsilon] d t\right. \\
\quad-\sum_{j=0}^{q-1} \int_{n T+\tau_{j}}^{n T+\tau_{j+1}} b(t) y^{T}(t) d t \\
\left.\quad-\int_{n T+\tau_{q}}^{(n+1) T} b(t) y^{T}(t) d t\right\} .
\end{aligned}
$$

Let $\epsilon \rightarrow 0$; we get the expression of $R_{0}$, which is given in (17). Therefore, if $R_{0}<1$, then $x(n T) \leq\left(R_{0}\right)^{n} x(0)$. Consequently, $x(n T) \rightarrow 0$ as $n \rightarrow \infty$.

Next, we can prove that $y(t) \rightarrow y^{T}(t)$ as $t \rightarrow \infty$. For any $\epsilon>0$, there must exist a $t_{1}>0$ such that $0<x(t)<\epsilon$ for $t>t_{1}$. Without loss of generality, we assume that $0<x(t)<\epsilon$ holds true for all $t>0$; then, we have

$$
\frac{d y(t)}{d t}<(\epsilon c(t)-\delta(t)) y(t)
$$

and consider the following impulsive differential equation:

$$
\begin{gathered}
\frac{d v(t)}{d t}=(\epsilon c(t)-\delta(t)) v(t), \quad t \neq \tau_{k}, \\
v\left(\tau_{k}^{+}\right)=v\left(\tau_{k}\right)+\sigma_{k}, \quad t=\tau_{k} .
\end{gathered}
$$

By using the same methods as those for system (23), it is easy to prove that system (29) has a globally stable periodic solution, denoted by $v^{T}(t)$ and

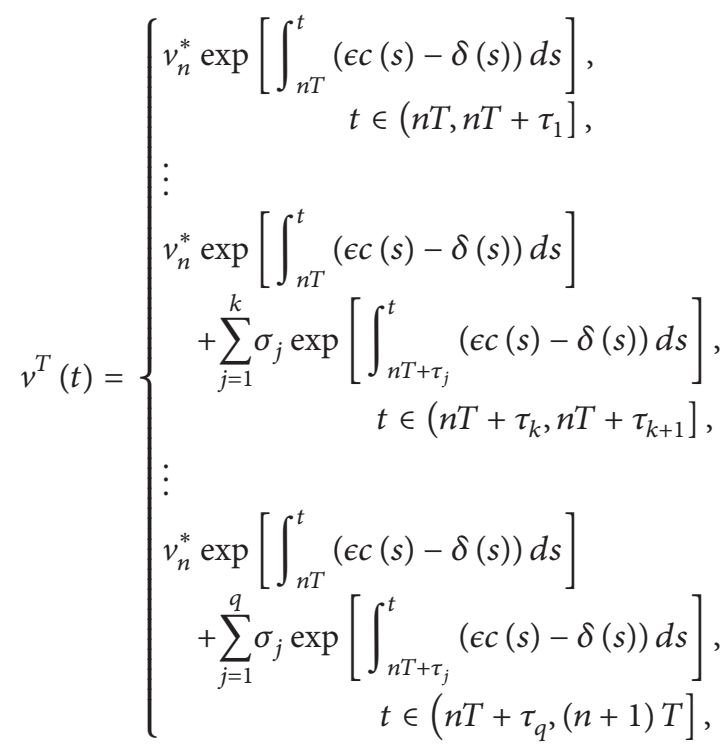

with

$$
v_{n}^{*}=\frac{\sum_{j=1}^{q} \sigma_{j} \exp \left[\int_{n T+\tau_{j}}^{(n+1) T}(\epsilon c(s)-\delta(s)) d s\right]}{1-\exp \left[\int_{n T}^{(n+1) T}(\epsilon c(s)-\delta(s)) d s\right]} .
$$

Therefore, combined with (24), for any $\epsilon_{1}>0$, there exists a $t_{2}>0$ such that $y^{T}(t)-\epsilon_{1}<y(t)<v^{T}(t)+\epsilon_{1}$ for any $t>t_{2}$. Let $\epsilon \rightarrow 0$; then we have $y^{T}(t)-\epsilon_{1}<y(t)<y^{T}(t)+\epsilon_{1}$ for $t>t_{2}$, which indicates that $y(t) \rightarrow y^{T}(t)$ as $t \rightarrow \infty$.

This completes the proof of global attractivity of $\left(0, y^{T}(t)\right)$. Then it is globally asymptotically stable. The proof of Theorem 2 is complete.

\section{The Optimal Control Time with Different $q$}

Assume that pesticide is sprayed and the natural enemy is released only at the time points $n T+\tau_{i}(i=1,2, \ldots, q$ and $0<\tau_{1}<\tau_{2}<\cdots<\tau_{q}<T$ ) during each period $T$. It is well known that the size of the pest population at the end will be different if impulsive control occurs at different time. So, it is necessary to determine the optimal time to make sure that the pest can be eliminated quickly.

3.1. The Optimal Control Time with $q=1$. In this subsection, we consider one-pulse controlling at time $n T+\tau_{1}$ in each period $T$ (where $\tau_{1} \in[0, T]$ ) with aims to find the optimal time $n T+\tau_{1}$ such that the threshold value is the smallest. 
Therefore, if $q=1$, then the threshold value $R_{0}$ can be reduced as

$$
\begin{aligned}
R_{0}=\left(1-p_{1}\right) & \\
& \times \exp \left[\int_{n T}^{(n+1) T} r(t) d t\right. \\
& -\int_{n T}^{n T+\tau_{1}} b(t) y^{T}(t) d t \\
& \left.-\int_{n T+\tau_{1}}^{(n+1) T} b(t) y^{T}(t) d t\right],
\end{aligned}
$$

where

$$
y^{T}(t)=\left\{\begin{array}{c}
Y_{n}^{*} \exp \left[-\int_{n T}^{t} \delta(s) d s\right], \\
t \in\left(n T, n T+\tau_{1}\right], \\
Y_{n}^{*} \exp \left[-\int_{n T}^{t} \delta(s) d s\right] \\
+\sigma_{1} \exp \left[-\int_{n T+\tau_{1}}^{t} \delta(s) d s\right], \\
t \in\left(n T+\tau_{1},(n+1) T\right], \\
Y_{n}^{*}=\frac{\sigma_{1} \exp \left[-\int_{n T+\tau_{1}}^{(n+1) T} \delta(s) d s\right]}{1-\exp \left[-\int_{n T}^{(n+1) T} \delta(s) d s\right]} .
\end{array}\right.
$$

Denote

$$
\begin{gathered}
A=\int_{n T}^{(n+1) T} r(t) d t \\
B\left(\tau_{1}\right)=Y_{n}^{*} \int_{n T}^{(n+1) T} b(t) \exp \left[-\int_{n T}^{t} \delta(s) d s\right] d t, \\
C_{1}\left(\tau_{1}\right)=\sigma_{1} \int_{n T+\tau_{1}}^{(n+1) T} b(t) \exp \left[-\int_{n T+\tau_{1}}^{t} \delta(s) d s\right] d t .
\end{gathered}
$$

Thus, we have

$$
R_{0}=\left(1-p_{1}\right) \exp \left(A-B\left(\tau_{1}\right)-C_{1}\left(\tau_{1}\right)\right) .
$$

Taking the derivative of function $R_{0}$ with respect to $\tau_{1}$, one obtains

$$
\begin{aligned}
\frac{d R_{0}}{d \tau_{1}}= & -\left(1-p_{1}\right)\left(\frac{d B\left(\tau_{1}\right)}{d \tau_{1}}+\frac{d C_{1}\left(\tau_{1}\right)}{d \tau_{1}}\right) \\
& \times \exp \left(A-B\left(\tau_{1}\right)-C_{1}\left(\tau_{1}\right)\right) \\
= & -R_{0}\left(\frac{d B\left(\tau_{1}\right)}{d \tau_{1}}+\frac{d C_{1}\left(\tau_{1}\right)}{d \tau_{1}}\right) \\
= & -R_{0}\left[\delta\left(n T+\tau_{1}\right)\left(B\left(\tau_{1}\right)+C_{1}\left(\tau_{1}\right)\right)-\sigma_{1} b\left(n T+\tau_{1}\right)\right] .
\end{aligned}
$$

Letting $d R_{0} / d \tau_{1}=0$, we can see that $\tau_{1 \text { min }}$ satisfies equation $d B\left(\tau_{1}\right) / d \tau_{1}+d C_{1}\left(\tau_{1}\right) / d \tau_{1}=0$; that is

$$
\begin{aligned}
G_{1} \doteq & \delta\left(n T+\tau_{1 \min }\right)\left(B\left(\tau_{1 \text { min }}\right)+C_{1}\left(\tau_{1 \text { min }}\right)\right) \\
& -\sigma_{1} b\left(n T+\tau_{1 \text { min }}\right)=0 .
\end{aligned}
$$

The second derivative of $R_{0}$ with respect to $\tau_{1}$ at $\tau_{1 \text { min }}$ can be calculated as follows:

$$
\begin{gathered}
\left.\frac{d^{2} R_{0}}{d \tau_{1}^{2}}\right|_{\tau_{1}=\tau_{1 \text { min }}} \\
=-R_{0}\left[\frac{d \delta\left(n T+\tau_{1}\right)}{d \tau_{1}}\left(B\left(\tau_{1 \text { min }}\right)+C_{1}\left(\tau_{1 \text { min }}\right)\right)\right. \\
\left.-\sigma_{1} \frac{d b\left(n T+\tau_{1 \mathrm{~min}}\right)}{d \tau_{1}}\right] \\
=-\sigma_{1} R_{0}\left[\left.\frac{b\left(n T+\tau_{1 \text { min }}\right)}{\delta\left(n T+\tau_{1 \text { min }}\right)} \frac{d \delta\left(n T+\tau_{1}\right)}{d \tau_{1}}\right|_{\tau_{1}=\tau_{1 \text { min }}}\right. \\
\left.\quad-\left.\frac{d b\left(n T+\tau_{1}\right)}{d \tau_{1}}\right|_{\tau_{1}=\tau_{1 \text { min }}}\right] .
\end{gathered}
$$

If $d^{2} R_{0} /\left.d \tau_{1}^{2}\right|_{\tau_{1}=\tau_{1 \min }}>0$, that is, $\tau_{1 \text { min }}$ satisfies

$$
\begin{gathered}
\left.\frac{b\left(n T+\tau_{1 \min }\right)}{\delta\left(n T+\tau_{1 \min }\right)} \frac{d \delta\left(n T+\tau_{1}\right)}{d \tau_{1}}\right|_{\tau_{1}=\tau_{1 \min }} \\
-\left.\frac{d b\left(n T+\tau_{1}\right)}{d \tau_{1}}\right|_{\tau_{1}=\tau_{1 \min }}
\end{gathered}
$$

$<0$,

then $\tau_{1 \mathrm{~min}}$ is the minimal value point.

According to the above discussion, we have the following theorem.

Theorem 3. If $\tau_{1 \text { min }}$ satisfies (37) and inequality (39), then the threshold value $R_{0}$ reaches its minimum value.

For example, if we let $r(t)=r_{0}+a \cos (\omega t), b(t)=$ $b_{0}+b \sin (\omega t)$, and $\delta(t)=\delta_{0}+\delta_{1} \cos (\omega t)$, then by simple calculations we have

$$
\begin{aligned}
& \left.\frac{d^{2} R_{0}}{d \tau_{1}^{2}}\right|_{\tau_{1}=\tau_{1 \text { min }}} \\
& \quad=\omega \sigma_{1} R_{0}\left[\frac{b \delta_{1}+\delta_{1} b_{0} \sin \left(\omega \tau_{1 \mathrm{~min}}\right)+b \delta_{0} \cos \left(\omega \tau_{1 \mathrm{~min}}\right)}{\delta_{0}+\delta_{1} \cos \left(\omega \tau_{1 \mathrm{~min}}\right)}\right],
\end{aligned}
$$

and if $\tau_{1 \text { min }}$ satisfies

$$
\frac{b \delta_{1}+\delta_{1} b_{0} \sin \left(\omega \tau_{1 \min }\right)+b \delta_{0} \cos \left(\omega \tau_{1 \mathrm{~min}}\right)}{\delta_{0}+\delta_{1} \cos \left(\omega \tau_{1 \mathrm{~min}}\right)}>0,
$$

then $\tau_{1 \text { min }}$ is the minimum value point. That is, $R_{0}$ reaches its minimum value when $\tau_{1}=\tau_{1 \text { min }}$.

To confirm our main results obtained in this subsection, we fixed all parameters including $r(t), a(t), b(t), c(t), \delta(t)$, $p_{1}, \sigma_{1}$, and initial values $x_{0}, y_{0}$ and carry out the numerical investigations. To find the optimal timing of applying IPM strategy, we consider $d R_{0} / d \tau_{1}$ as a function with respect to $\tau_{1}$ aiming to find the time point such that $d R_{0} / d \tau_{1}=0$. 


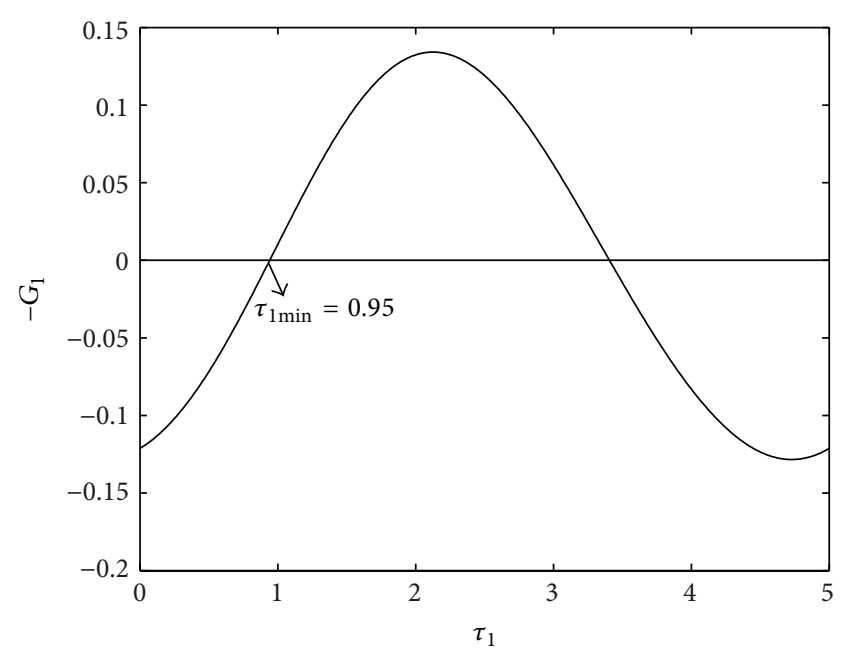

FIGURE 1: Illustration of the existence of the minimal value point. The parameter values are fixed as follows: $r(t)=1.5+0.3 \cos (0.4 \pi t)$, $b(t)=1+0.2 \sin (0.4 \pi t), \delta(t)=0.5+0.1 \cos (0.4 \pi t), \sigma_{1}=0.5$, and $p_{1}=0.25$.

Since $d R_{0} / d \tau_{1}=-R_{0} G_{1}=0$, we only need to plot the $-G_{1}$ with respect to time $\tau_{1}$, as shown in Figure 1. By calculation, we have $\tau_{1 \mathrm{~min}}=0.95$, which satisfies inequality (41), and consequently $\tau_{1 \text { min }}$ is a minimum value point.

Now, we can consider the effects of different timing of applying IPM strategies on the pest population, in particular the amplitudes of the pest population. To do this, we choose three different time points, denoted by $\tau_{1 \text { min }}, \tau_{1}$, and $\tau_{1}^{\prime}$, at which the one-time control action has been implemented. It follows from Figure 2 that the maximal value of the pest population is the smallest when we implement the one-time control action at time $\tau_{1 \mathrm{~min}}$, which confirms that the results obtained here can help us to design the optimal control strategies.

In the following, we would like to address how the impulsive period $T$, release quantity $\sigma_{1}$, pest killing rate $p_{1}$, and death rate of the pest population $b(t)$ affect the threshold value $R_{0}$. To address this question, we fix the parameters concerning periodic functions $r(t), a(t), c(t)$, and $\delta(t)$ and vary the impulsive period $T$, the release quantity $\sigma_{1}$, the killing rate $p_{1}$, and the death rate $b(t)$, respectively.

In Figure 3, we see that the threshold value $R_{0}$ is not monotonic with respect to time $t$ and the effects of all four parameters $\left(T, \sigma_{1}, p_{1}\right.$, and $\left.b_{0}\right)$ on threshold condition $R_{0}$ are complex. Figure 3(a) shows the effect of impulsive period $T$ on the $R_{0}$, and the results indicate that the larger the impulsive period $T$ is, the larger the threshold value is, which will result in a more sever pest outbreak. Oppositely, in Figure 3(b), the results indicate that the smaller the release quantity $\sigma_{1}$ is, the larger the $R_{0}$ is. Figures 3(c) and 3(d) clarify that slightly increasing the pest killing rate $p_{1}$ and the death rate of pest $b(t)$ can reduce the quantity of threshold value $R_{0}$ dramatically, and the results can be used to help the farmer to select appropriate pesticides. At the same time, we can see clearly how the periodic perturbations affect the threshold value $R_{0}$, as indicated in the bold curves in Figure 3 .
3.2. The Optimal Control Time with $q=2$. In this subsection, we consider two-pulse controlling at times $n T+\tau_{1}$ and $n T+\tau_{2}$ in each period $T$, where $0<\tau_{1}<\tau_{2}<T$. In the following, we focus on finding the optimal time points $n T+\tau_{1}$ and $n T+\tau_{2}$ such that the threshold value $R_{0}$ is the smallest.

Therefore, if $q=2$, then the threshold value $R_{0}$ becomes as

$$
\begin{aligned}
R_{0}=\left(1-p_{1}\right) & \left(1-p_{2}\right) \\
\times \exp \left[\int_{n T}^{(n+1) T} r(t) d t\right. & \\
& -\int_{n T}^{n T+\tau_{1}} b(t) y^{T}(t) d t \\
& -\int_{n T+\tau_{1}}^{n T+\tau_{2}} b(t) y^{T}(t) d t \\
& \left.-\int_{n T+\tau_{2}}^{(n+1) T} b(t) y^{T}(t) d t\right],
\end{aligned}
$$

where

$$
y^{T}(t)=\left\{\begin{array}{r}
Y_{n}^{*} \exp \left[-\int_{n T}^{t} \delta(s) d s\right], \\
t \in\left(n T, n T+\tau_{1}\right], \\
Y_{n}^{*} \exp \left[-\int_{n T}^{t} \delta(s) d s\right] \\
+\sigma_{1} \exp \left[-\int_{n T+\tau_{1}}^{t} \delta(s) d s\right] \\
t \in\left(n T+\tau_{1}, n T+\tau_{2}\right] \\
Y_{n}^{*} \exp \left[-\int_{n T}^{t} \delta(s) d s\right] \\
+\sigma_{1} \exp \left[-\int_{n T+\tau_{1}}^{t} \delta(s) d s\right] \\
+\sigma_{2} \exp \left[-\int_{n T+\tau_{2}}^{t} \delta(s) d s\right] \\
Y_{n}^{*}=\frac{\sigma_{1} \exp \left[-\int_{n T+\tau_{1}}^{(n+1) T} \delta(s) d s\right]}{1-\exp \left[-\int_{n T}^{(n+1) T} \delta(s) d s\right]} \\
+\frac{\sigma_{2}^{*} \exp \left[-\int_{n T+\tau_{2}}^{(n+1) T} \delta(s) d s\right]}{1-\exp \left[-\int_{n T}^{(n+1) T} \delta(s) d s\right]} \\
\left.+Y_{n_{2}}^{*} \cdot(n+1) T\right]
\end{array}\right.
$$

Thus, we get

$$
\begin{aligned}
R_{0}= & \left(1-p_{1}\right)\left(1-p_{2}\right) \\
& \times \exp \left(A-B\left(\tau_{1}, \tau_{2}\right)-C_{1}\left(\tau_{1}\right)-C_{2}\left(\tau_{2}\right)\right),
\end{aligned}
$$

where

$$
\begin{gathered}
A=\int_{n T}^{(n+1) T} r(t) d t \\
C_{1}\left(\tau_{1}\right)=\sigma_{1} \int_{n T+\tau_{1}}^{(n+1) T} b(t) \exp \left[-\int_{n T+\tau_{1}}^{t} \delta(s) d s\right] d t
\end{gathered}
$$




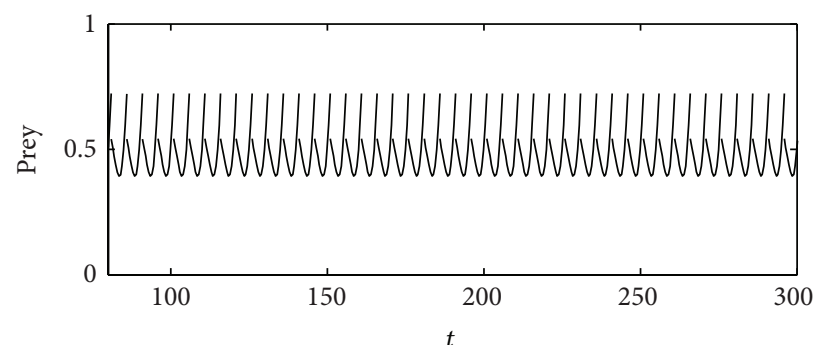

(a)

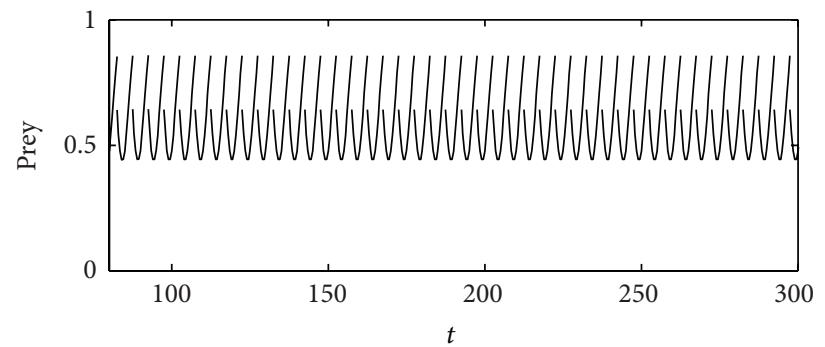

(c)

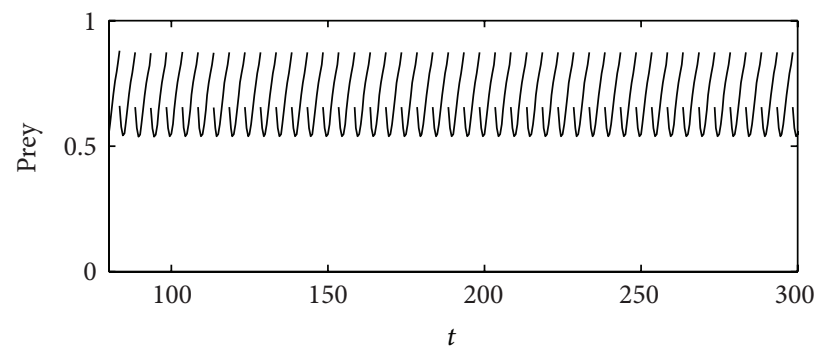

(e)

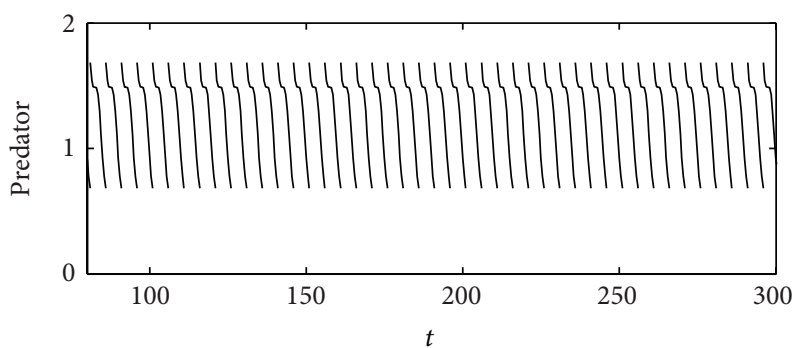

(b)

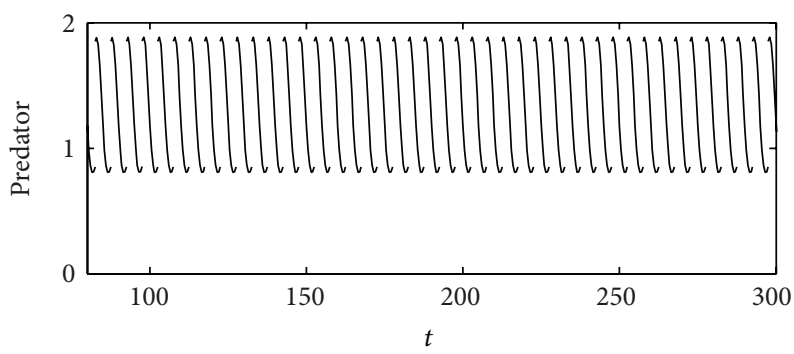

(d)

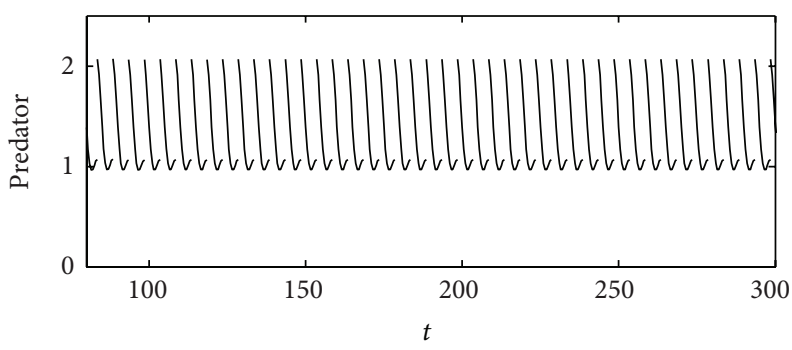

(f)

FIGURE 2: Supposing that one-pulse controlling is implemented in each period $T$, we plot the solution curve of system (4). We fixed all parameters as follows: $r(t)=0.7+0.1 \cos (0.4 \pi t), a(t)=1 /(50+9 \sin (0.4 \pi t)), b(t)=0.5+0.02 \sin (0.4 \pi t), c(t)=0.5 b(t), \delta(t)=0.3+$ $0.2 \cos (0.4 \pi t), p_{1}=0.25, \sigma_{1}=1, x_{0}=2.6$, and $y_{0}=1$. The impulsive timing is $(\mathrm{a}-\mathrm{b}) \tau_{1 \min }=0.95 ;(\mathrm{c}-\mathrm{d}) \tau_{1}=2.5 ;(\mathrm{e}-\mathrm{f}) \tau_{1}^{\prime}=3.5$.

$$
\begin{aligned}
C_{2}\left(\tau_{2}\right) & =\sigma_{2} \int_{n T+\tau_{2}}^{(n+1) T} b(t) \exp \left[-\int_{n T+\tau_{2}}^{t} \delta(s) d s\right] d t \\
B\left(\tau_{1}, \tau_{2}\right) & \\
= & Y_{n}^{*} \int_{n T}^{(n+1) T} b(t) \exp \left[-\int_{n T}^{t} \delta(s) d s\right] d t \\
= & Y_{n_{1}}^{*} \int_{n T}^{(n+1) T} b(t) \exp \left[-\int_{n T}^{t} \delta(s) d s\right] d t \\
& +Y_{n_{2}}^{*} \int_{n T}^{(n+1) T} b(t) \exp \left[-\int_{n T}^{t} \delta(s) d s\right] d t \\
\doteq & B_{1}\left(\tau_{1}\right)+B_{2}\left(\tau_{2}\right) .
\end{aligned}
$$

Taking the derivatives of the function $R_{0}$ with respect to $\tau_{1}$ and $\tau_{2}$, respectively, we get

$$
\begin{aligned}
& \frac{\partial R_{0}}{\partial \tau_{1}}=-R_{0}\left(\frac{d B_{1}\left(\tau_{1}\right)}{d \tau_{1}}+\frac{d C_{1}\left(\tau_{1}\right)}{d \tau_{1}}\right), \\
& \frac{\partial R_{0}}{\partial \tau_{2}}=-R_{0}\left(\frac{d B_{2}\left(\tau_{2}\right)}{d \tau_{2}}+\frac{d C_{2}\left(\tau_{2}\right)}{d \tau_{2}}\right),
\end{aligned}
$$

with

$$
\begin{gathered}
\frac{d B_{i}\left(\tau_{i}\right)}{d \tau_{i}}=\delta\left(n T+\tau_{i}\right) B_{i}\left(\tau_{i}\right), \quad(i=1,2), \\
\frac{d C_{i}\left(\tau_{i}\right)}{d \tau_{i}}=\delta\left(n T+\tau_{i}\right) C_{i}\left(\tau_{i}\right)-\sigma_{i} b\left(n T+\tau_{i}\right), \quad(i=1,2) .
\end{gathered}
$$

Letting $\partial R_{0} / \partial \tau_{1}=0, \partial R_{0} / \partial \tau_{2}=0$, we have $\tau_{1 \text { min }}$ and $\tau_{2 \min }$ satisfying the following equations $d B_{i}\left(\tau_{i}\right) / d \tau_{i}+$ $d C_{i}\left(\tau_{i}\right) / d \tau_{i}=0$; that is,

$$
\begin{array}{r}
G_{i} \doteq \delta\left(n T+\tau_{i}\right)\left(B_{i}\left(\tau_{i}\right)+C_{i}\left(\tau_{i}\right)\right)-\sigma_{i} b\left(n T+\tau_{i}\right)=0, \\
(i=1,2) .
\end{array}
$$

The second partial derivatives of the function $R_{0}$ with respect to $\tau_{1}, \tau_{2}$ at the points $\tau_{1 \text { min }}$ and $\tau_{2 \text { min }}$ can be calculated as follows:

$$
\begin{aligned}
R_{11}^{\prime \prime} & =\left.\frac{\partial^{2} R_{0}}{\partial \tau_{1}^{2}}\right|_{\tau_{1}=\tau_{\min }} \\
& =-\sigma_{1} R_{0}\left[\left.\frac{b\left(n T+\tau_{1 \min }\right)}{\delta\left(n T+\tau_{1 \min }\right)} \frac{d \delta\left(n T+\tau_{1}\right)}{d \tau_{1}}\right|_{\tau_{1}=\tau_{1 \min }}\right.
\end{aligned}
$$



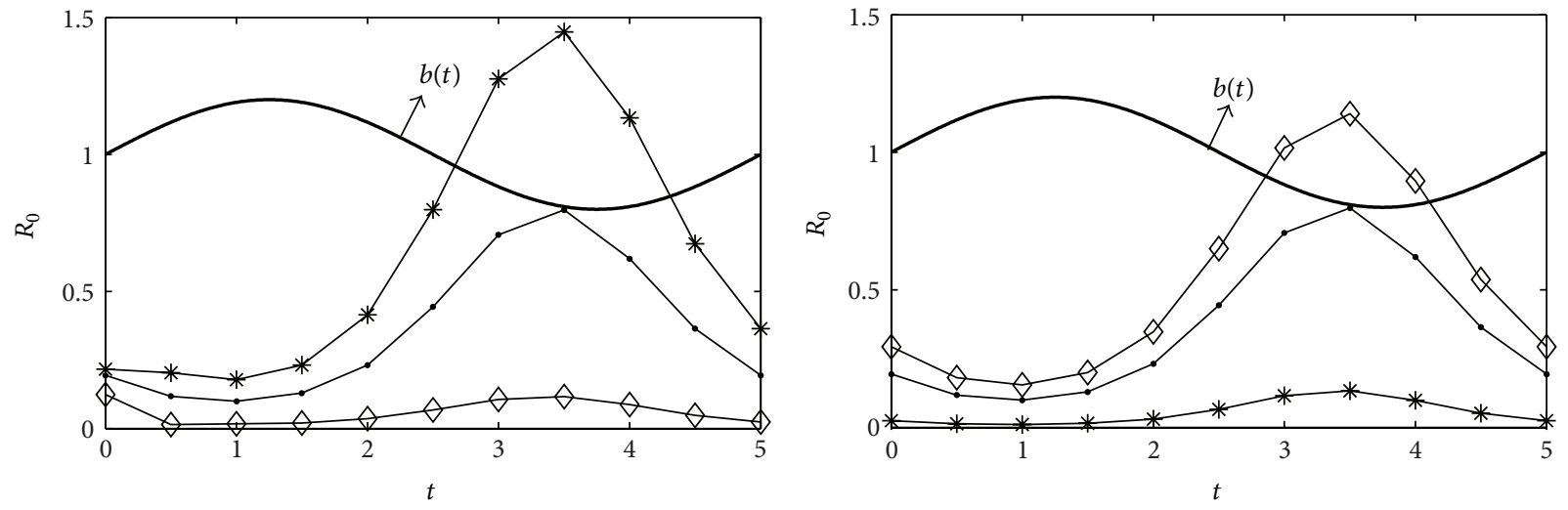

$$
\begin{aligned}
& \diamond T=4.8 \\
& \multimap T=5 \\
& * \quad T=5.05
\end{aligned}
$$

(a)

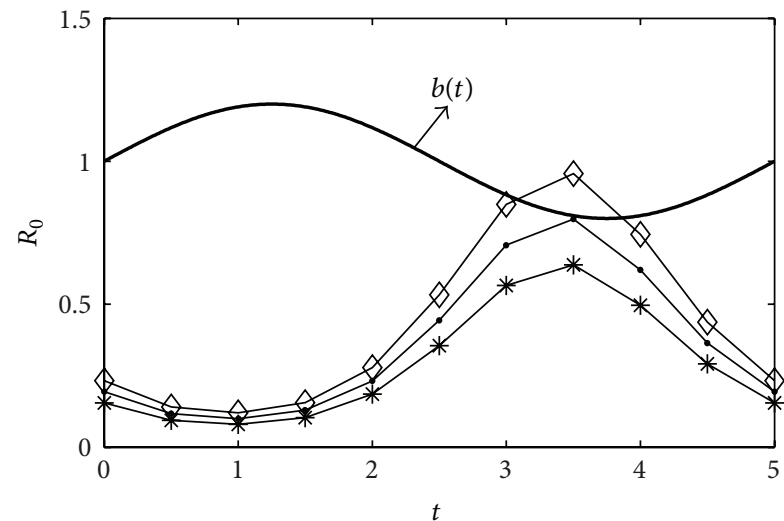

$$
\begin{aligned}
& \diamond p_{1}=0.1 \\
& \rightarrow p_{1}=0.25 \\
& * p_{1}=0.4
\end{aligned}
$$

(c)

$$
\begin{aligned}
& \diamond \sigma_{1}=4.5 \\
& \rightarrow \sigma_{1}=5 \\
& * \sigma_{1}=6
\end{aligned}
$$

(b)

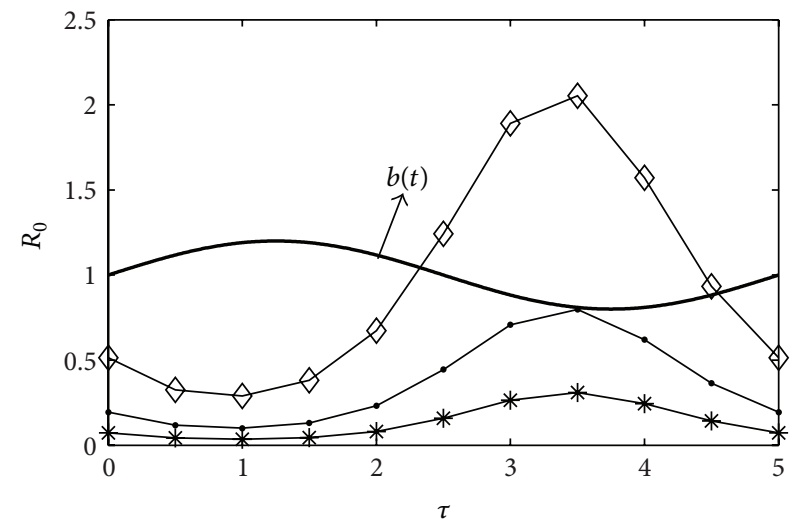

$$
\begin{aligned}
& \diamond b_{0}=0.9 \\
& -b_{0}=1 \\
& \rightarrow b_{0}=1.1
\end{aligned}
$$

(d)

FIGURE 3: The effects of the impulsive period $T$, the releasing quantity $\sigma_{1}$, the killing rate $p_{1}$, and the death rate $b(t)$ on the threshold condition $R_{0}$. The baseline parameter values are as follows: $r(t)=1.8+0.3 \cos (0.4 \pi t), b(t)=1+0.2 \sin (0.4 \pi t), \delta(t)=0.5+0.1 \cos (0.4 \pi t), p_{1}=0.25$, $T=5, \sigma_{1}=5$. (a) The effect of the period $T$ on $R_{0}$; (b) the effect of the releasing quantity $\sigma_{1}$ on $R_{0}$; (c) the effect of pest killing rate $p_{1}$ on $R_{0}$; (d) the effect of death rate $b_{0}$ on $R_{0}$.

$$
\begin{aligned}
& \left.-\left.\frac{d b\left(n T+\tau_{1}\right)}{d \tau_{1}}\right|_{\tau_{1}=\tau_{1 \min }}\right] \\
& R_{22}^{\prime \prime}=\left.\frac{\partial^{2} R_{0}}{\partial \tau_{2}^{2}}\right|_{\tau_{2}=\tau_{2 \min }} \\
& =-\sigma_{2} R_{0}\left[\left.\frac{b\left(n T+\tau_{2 \min }\right)}{\delta\left(n T+\tau_{2 \min }\right)} \frac{d \delta\left(n T+\tau_{2}\right)}{d \tau_{2}}\right|_{\tau_{2}=\tau_{2 \min }}\right. \\
& \left.-\left.\frac{d b\left(n T+\tau_{2}\right)}{d \tau_{2}}\right|_{\tau_{2}=\tau_{2 \min }}\right] \\
& \begin{aligned}
R_{12}^{\prime \prime} & =\left.\frac{\partial^{2} R_{0}}{\partial \tau_{1} \partial \tau_{2}}\right|_{\left(\tau_{1}=\tau_{1 \min }, \tau_{2}=\tau_{2 \min }\right)} \\
& =-\frac{\partial R_{0}}{\partial \tau_{2}}\left(\frac{d B_{1}\left(\tau_{1}\right)}{d \tau_{1}}+\frac{d C_{1}\left(\tau_{1}\right)}{d \tau_{1}}\right)=0 .
\end{aligned} \\
& R_{11}^{\prime \prime} R_{22}^{\prime \prime}-\left(R_{12}^{\prime \prime}\right)^{2} \\
& =\sigma_{1} \sigma_{2} R_{0}^{2} \prod_{i=1}^{2}\left[\left.\frac{b\left(n T+\tau_{i \min }\right)}{\delta\left(n T+\tau_{i \min }\right)} \frac{d \delta\left(n T+\tau_{i}\right)}{d \tau_{i}}\right|_{\tau_{i}=\tau_{i \min }}\right. \\
& \left.-\left.\frac{d b\left(n T+\tau_{i}\right)}{d \tau_{i}}\right|_{\tau_{i}=\tau_{i \min }}\right] \text {. }
\end{aligned}
$$




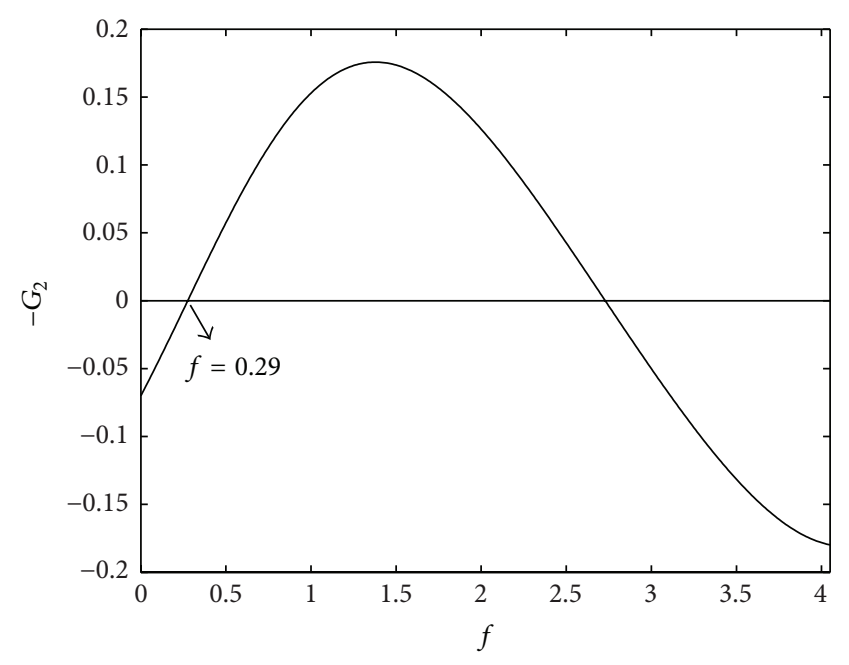

FIGURE 4: Illustrations of existences of $\tau_{i \min }(i=1,2)$. The parameter values are fixed as follows: $b(t)=1+0.2 \sin (0.7 \pi t), \delta(t)=0.5+$ $0.1 \cos (0.7 \pi t), \sigma_{1}=0.5$.

According to the method of extremes for multivariable function, if $R_{11}^{\prime \prime} R_{22}^{\prime \prime}-\left(R_{12}^{\prime \prime}\right)^{2}>0$, and $R_{11}^{\prime \prime}>0$, that is, if $\tau_{i \text { min }}(i=1,2)$ satisfy

$$
\begin{aligned}
& \left.\frac{b\left(n T+\tau_{i \min }\right)}{\delta\left(n T+\tau_{i \min }\right)} \frac{d \delta\left(n T+\tau_{i}\right)}{d \tau_{i}}\right|_{\tau_{i}=\tau_{\min }} \\
& -\left.\frac{d b\left(n T+\tau_{i}\right)}{d \tau_{i}}\right|_{\tau_{i}=\tau_{i \min }}<0, \quad(i=1,2),
\end{aligned}
$$

then $\tau_{i \min }(i=1,2)$ are the minimum value points.

From the above argument, we have the following theorem.

Theorem 4. If $\tau_{i \min }(i=1,2)$ satisfy (48) and inequalities (51), then threshold value $R_{0}$ reaches its minimum value.

Similarly, in order to get the exact values for $\tau_{i \min }$, we let $r(t)=r_{0}+a \cos (\omega t), b(t)=b_{0}+b \sin (\omega t), \delta(t)=$ $\delta_{0}+\delta_{1} \cos (\omega t)$; then,

$$
\begin{aligned}
G_{i}= & {\left[\delta_{0}+\delta_{1} \cos \left(\omega \tau_{i}\right)\right]\left[B_{i}\left(\tau_{i}\right)+C_{i}\left(\tau_{i}\right)\right] } \\
& -\sigma_{i}\left[b_{0}+b \sin \left(\omega \tau_{i}\right)\right], \quad(i=1,2)
\end{aligned}
$$

and if $\tau_{i \min }(i=1,2)$ satisfy

$$
\frac{b \delta_{1}+\delta_{1} b_{0} \sin \left(\omega \tau_{i \min }\right)+b \delta_{0} \cos \left(\omega \tau_{i \min }\right)}{\delta_{0}+\delta_{1} \cos \left(\omega \tau_{i \min }\right)}>0,
$$

then $\tau_{i \text { min }}(i=1,2)$ are the minimum value points. Since $\tau_{2}>$ $\tau_{1}$, we may assume that $\tau_{2}=\tau_{1}+f$, where $f$ is a positive constant. Then, we plot $-G_{2}$ with respect to $f$, as shown in Figure 4. It is clear that the minimum value point of $f$ is about 0.29 . Thus, $\tau_{2 \min }=\tau_{1 \min }+0.29=1.24$.

In the following, we will discuss the effects of the pest killing rates $\left(p_{1}, p_{2}\right)$ and releasing quantities $\left(\sigma_{1}, \sigma_{2}\right)$ on the threshold value $R_{0}$. As we can see from Figure $5, R_{0}$ is quite sensitive to all four parameters $\left(p_{1}, p_{2}, \sigma_{1}, \sigma_{2}\right)$. According to these numerical simulations, the farmer can take appropriate measures to achieve successful pest control.

Moreover, we can investigate the more general case, that is, $q$-time impulsive control actions implemented at time $n T+$ $\tau_{i}\left(i=1,2, \ldots, q\right.$ and $\left.\tau_{1}<\tau_{2}<\cdots<\tau_{q}\right)$ within period $(n T,(n+1) T]$ [22]. Similarly, we can get a unique group of optimal impulsive moments for $i=1,2, \ldots q$, which satisfy

$$
\begin{array}{r}
G_{i} \doteq \delta\left(n T+\tau_{i}\right)\left(B_{i}\left(\tau_{i}\right)+C_{i}\left(\tau_{i}\right)\right)-\sigma_{i} b\left(n T+\tau_{i}\right)=0, \\
(i=1,2, \ldots q) .
\end{array}
$$

In fact,

$$
\begin{aligned}
R_{0}= & \prod_{i=1}^{q}\left(1-p_{i}\right) \\
& \times \exp \left(A-B\left(\tau_{1}, \tau_{2}, \ldots, \tau_{q}\right)-C\left(\tau_{1}, \tau_{2}, \ldots, \tau_{q}\right)\right),
\end{aligned}
$$

where

$$
\begin{aligned}
B\left(\tau_{1}, \tau_{2}, \ldots, \tau_{q}\right)= & \sum_{j=1}^{q} Y_{n_{j}}^{*} \int_{n T}^{(n+1) T} b(t) \exp \left[-\int_{n T}^{t} \delta(s) d s\right] d t \\
\doteq & \sum_{j=1}^{q} B_{i}\left(\tau_{i}\right) \\
C\left(\tau_{1}, \tau_{2}, \ldots, \tau_{q}\right)= & \sum_{j=1}^{q} \sigma_{j} \int_{n T+\tau_{j}}^{(n+1) T} b(t) \\
& \times \exp \left[-\int_{n T+\tau_{j}}^{t} \delta(s) d s\right] d t \\
& \doteq \sum_{j=1}^{q} C_{i}\left(\tau_{i}\right) .
\end{aligned}
$$

Taking the derivatives of the function $R_{0}$ with respect to $\tau_{i}(i=1,2, \ldots, q)$, respectively, we get

$$
\begin{array}{r}
\frac{\partial R_{0}}{\partial \tau_{i}}=-R_{0}\left(\frac{d B_{i}\left(\tau_{i}\right)}{d \tau_{i}}+\frac{d C_{i}\left(\tau_{i}\right)}{d \tau_{i}}\right), \\
(i=1,2, \ldots, q)
\end{array}
$$

with

$$
\begin{gathered}
\frac{d B_{i}\left(\tau_{i}\right)}{d \tau_{i}}=\delta\left(n T+\tau_{i}\right) B_{i}\left(\tau_{i}\right), \\
\frac{d C_{i}\left(\tau_{i}\right)}{d \tau_{i}}=\delta\left(n T+\tau_{i}\right) C_{i}\left(\tau_{i}\right)-\sigma_{i} b\left(n T+\tau_{i}\right) . \\
(i=1,2, \ldots, q) .
\end{gathered}
$$

Letting $\partial R_{0} / \partial \tau_{i}=0,(i=1,2, \ldots, q)$, we have $\tau_{i \text { min }}$ satisfying (54). 


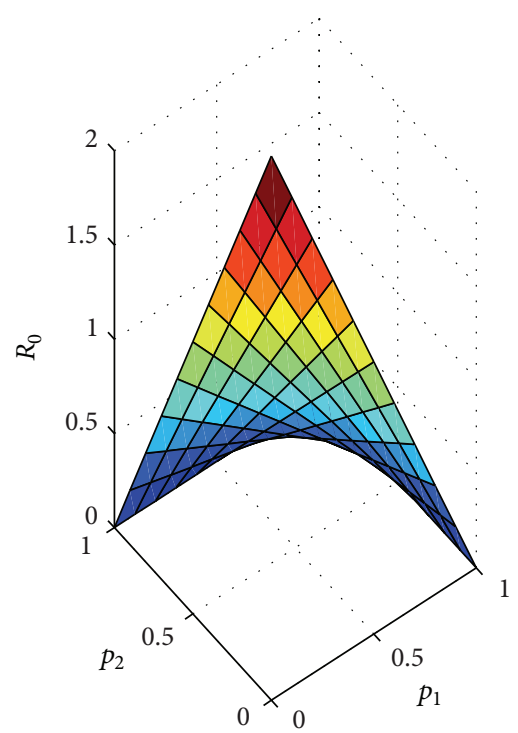

(a)

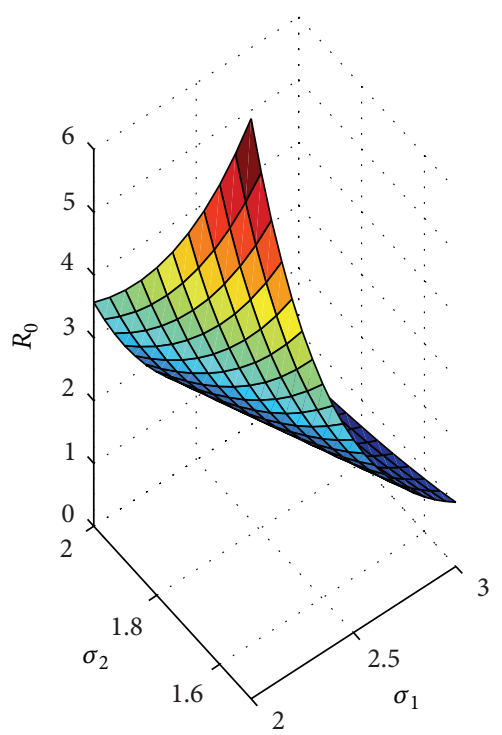

(b)

Figure 5: The effects of the pest killing rates $\left(p_{1}, p_{2}\right)$ and the releasing quantities $\left(\sigma_{1}, \sigma_{2}\right)$ on the threshold condition $R_{0}$. The baseline parameter values are as follows: $r(t)=2+0.3 \cos (0.4 \pi t), b(t)=1+0.2 \sin (0.4 \pi t), \delta(t)=0.5+0.1 \cos (0.4 \pi t), T=5, \tau_{1}=1$, $\tau_{2}=3$. (a) The effect of the releasing quantities $p_{1}$ and $p_{2}$ on $R_{0}$ with $\sigma_{1}=2.5$ and $\sigma_{2}=1.8$; (b) the effect of pest killing rates $\sigma_{1}$ and $\sigma_{2}$ on $R_{0}$ with $p_{1}=0.25$ and $p_{2}=0.2$.

If $r(t)=r_{0}+a \cos (\omega t), b(t)=b_{0}+b \sin (\omega t)$, and $\delta(t)=$ $\delta_{0}+\delta_{1} \cos (\omega t)$, then

$$
\begin{aligned}
G_{i}= & {\left[\delta_{0}+\delta_{1} \cos \left(\omega \tau_{i}\right)\right]\left[B_{i}\left(\tau_{i}\right)+C_{i}\left(\tau_{i}\right)\right] } \\
& -\sigma_{i}\left[b_{0}+b \sin \left(\omega \tau_{i}\right)\right], \quad(i=1,2, \ldots, q) .
\end{aligned}
$$

This indicates that if $\tau_{i \min }(i=1,2, \ldots, q)$ satisfy the following inequalities:

$$
\begin{array}{r}
\frac{b \delta_{1}+\delta_{1} b_{0} \sin \left(\omega \tau_{i \min }\right)+b \delta_{0} \cos \left(\omega \tau_{i \min }\right)}{\delta_{0}+\delta_{1} \cos \left(\omega \tau_{i \min }\right)}>0, \\
(i=1,2, \ldots, q),
\end{array}
$$

then, $\tau_{i \min }(i=1,2, \ldots, q)$ are the minimum value points.

\section{Discussion and Biological Conclusions}

It is well known that the growth rate of the species is affected by the living environments, so it is more practical to consider the growth rates of predator and prey as the functions with respect to time $t$ in the models with IPM strategies. Therefore, nonautonomous predator-prey systems with impulsive effects have been developed and investigated in the literatures [11-13]. However, those works mainly focused on the dynamical behavior including the existence and stability of pest-free periodic solutions. From pest control point of view, one of interesting questions is to determine the optimal application timing of pest control tactics in such models and fall within the scope of the study.

In order to address this question, the existence and global stability of pest-free periodic solution have been proved in theory firstly. Moreover, the optimal application timings which minimize the threshold value for one-time pulse control, two-time pulse controls, and multipulse controls within a given period have been obtained, and most importantly the analytical formula of the optimal timings of IPM applications has been provided for each case. For examples, Figure 1 illustrates the existence of the minimum value point of the threshold value $R_{0}$, under which the maximum amplitude of pest population reaches its minimum value, and this is validated by comparison of different sizes of pest population at three different impulsive timings in Figure 2; Figure 3 clarifies how the four parameters (the impulsive period, the release quantity, the killing rate of pest, and the death rate of pest) affect the successful pest control. All those results obtained here are useful for the farmer to select appropriate timings at which the IPM strategies are applied.

Note that the complex dynamical behavior of model (4) can be seen in Figure 6, where the period $T$ is chosen as a bifurcation parameter. As the parameter $T$ increases, model (4) may give different solutions with period $T, 2 T$, and eventually the model (4) undergoes a period-double bifurcation and leads to chaos. Moreover, the multiple attractors can coexist for a wide range of parameter, which indicates that the final stable states of pest and natural enemy populations depend on their initial densities.

In this paper, we mainly focus on the simplest preypredator model with impulsive effects. It is interesting to consider the evolution of pesticide resistance, which can be involved into the killing rate related to pesticide applications. Moreover, according to the definition of IPM, the control actions can only be applied once the density of pest populations reaches the economic threshold. Therefore, based on above facts, we would like to develop more realistic models in our future works. 


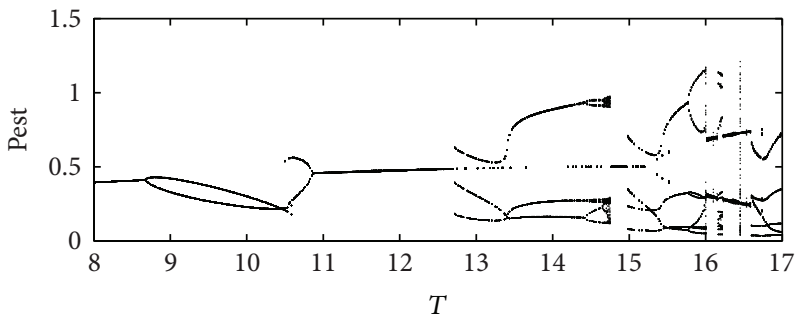

(a)

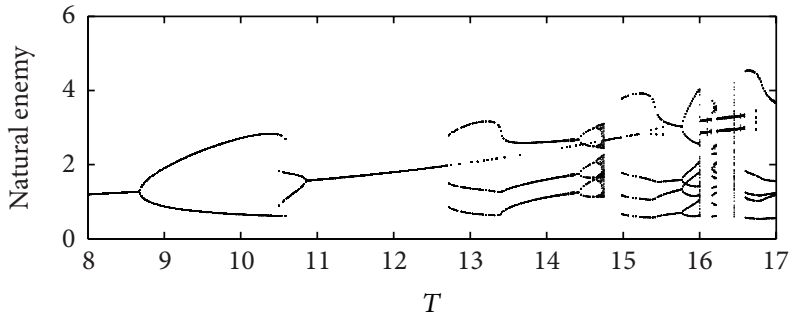

(b)

Figure 6: The bifurcation diagram of stable periodic solution of system (1) with $a(t)=1 /(50+15 \sin (3 t)), b(t)=0.5, c(t)=0.5, \delta(t)=0.4$, $r(t)=0.5, p_{1}=0.7, \sigma_{1}=0.5$. (a) The bifurcation diagram of pest for system (1); (b) the bifurcation diagram of natural enemy for system (1).

\section{Conflict of Interests}

The authors declare that there is no conflict of interests regarding the publication of this paper.

\section{Acknowledgments}

This work is supported by the National Natural Science Foundation of China (NSFCs, 11171199, 11371030, 11301320) and the Fundamental Research Funds for the Central Universities (GK201305010, GK201401004, and GK201402007).

\section{References}

[1] M. L. Flint, "Integrated Pest Management for Walnuts, seconded. University of California Statewide Integrated Pest Management Project," Division of Agriculture and Natural Resources, University of California, Oakland, Calif, USA, publication 3270, pp. 3641.

[2] J. C. Van Lenteren, "Integrated pest management in protected crops," in Integrated Pest Management, D. Dent, Ed., pp. 311-343, Chapman and Hall, London, UK, 1995.

[3] J. C. Van Lenteren, "Success in biological control of arthropods by augmentation of natural enemies," in Biological Control: Measures of Success, pp. 77-103, 2000.

[4] J. C. Van Lenteren and J. Woets, "Biological and integrated pest control in greenhouses," Annual Review of Entomology, vol. 33, pp. 239-269, 1988.

[5] C. Li and S. Tang, "The effects of timing of pulse spraying and releasing periods on dynamics of generalized predator-prey model," International Journal of Biomathematics, vol. 5, Article ID 1250012, 27 pages, 2012.

[6] W. Gao and S. Tang, "The effects of impulsive releasing methods of natural enemies on pest control and dynamical complexity," Nonlinear Analysis: Hybrid Systems, vol. 5, no. 3, pp. 540-553, 2011.

[7] S. Tang, G. Tang, and R. A. Cheke, "Optimum timing for integrated pest management: modelling rates of pesticide application and natural enemy releases," Journal of Theoretical Biology, vol. 264, no. 2, pp. 623-638, 2010.

[8] J. Liang and S. Tang, "Optimal dosage and economic threshold of multiple pesticide applications for pest control," Mathematical and Computer Modelling, vol. 51, no. 5-6, pp. 487-503, 2010.
[9] X. Liu and L. Chen, "Complex dynamics of Holling type II Lotka-Volterra predator-prey system with impulsive perturbations on the predator," Chaos, Solitons and Fractals, vol. 16, no. 2, pp. 311-320, 2003.

[10] S. Y. Tang and L. S. Chen, "The periodic predator-prey LotkaVolterra model with impulsive effect," Journal of Mechanics in Medicine and Biology, vol. 2, no. 3-4, pp. 267-296, 2002.

[11] S. Tang, Y. Xiao, L. Chen, and R. A. Cheke, "Integrated pest management models and their dynamical behaviour," Bulletin of Mathematical Biology, vol. 67, no. 1, pp. 115-135, 2005.

[12] S. Tang and R. A. Cheke, "Models for integrated pest control and their biological implications," Mathematical Biosciences, vol. 215, no. 1, pp. 115-125, 2008.

[13] L. Mailleret and F. Grognard, "Global stability and optimisation of a general impulsive biological control model," Mathematical Biosciences, vol. 221, no. 2, pp. 91-100, 2009.

[14] S. Tang and L. Chen, "Modelling and analysis of integrated pest management strategy," Discrete and Continuous Dynamical Systems B: A Journal Bridging Mathematics and Sciences, vol. 4, no. 3, pp. 759-768, 2004.

[15] S. Y. Tang and Y. N. Xiao, Biological Dynamics of Single Species, Science Press, 2008 (Chinese).

[16] H. M. Safuan, Z. Jovanoski, I. N. Towers, and H. S. Sidhu, "Exact solution of a non-autonomous logistic population model," Ecological Modelling, vol. 251, pp. 99-102, 2013.

[17] X. Meng and L. Chen, "Almost periodic solution of nonautonomous Lotka-Volterra predator-prey dispersal system with delays," Journal of Theoretical Biology, vol. 243, no. 4, pp. 562-574, 2006.

[18] F. F. Zhang and S. Y. Tang, "Dynamical behaviour of periodic impulsive chemical control model with periodically switching pesticides," Mathematical Biology, vol. 2, pp. 497-502, 2011.

[19] V. Lakshmikantham, D. D. Bainov, and P. S. Simeonov, Theory of Impulsive Differential Equations, World Scientific, Singapore, 1989.

[20] D. D. Bainov and P. S. Simenov, Systems with Impulsive Effect: Stability the-Ory and Applications, Ellis Horwood, Chichester, UK, 1989.

[21] D. Baĭnov and P. Simeonov, Impulsive Differential Equations: Periodic Solutions and Applications, vol. 66 of Pitman Monographs and Surveys in Pure and Applied Mathematics, 1993.

[22] Y. Xue, S. Tang, and J. Liang, "Optimal timing of interventions in fishery resource and pest management," Nonlinear Analysis: Real World Applications, vol. 13, no. 4, pp. 1630-1646, 2012. 


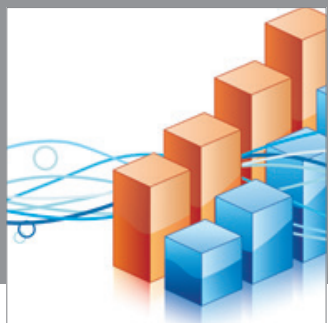

Advances in

Operations Research

mansans

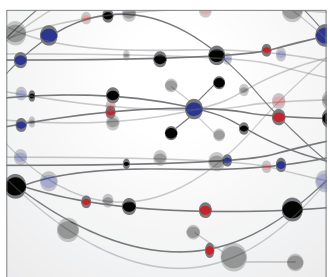

The Scientific World Journal
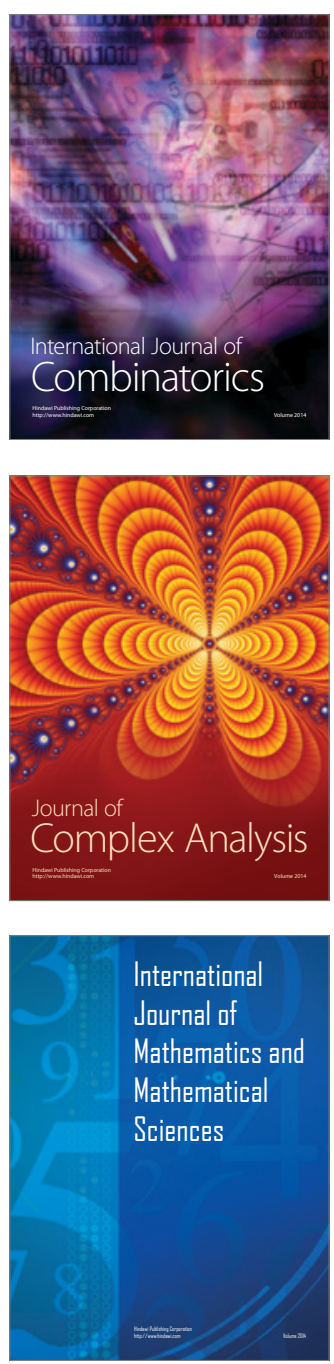
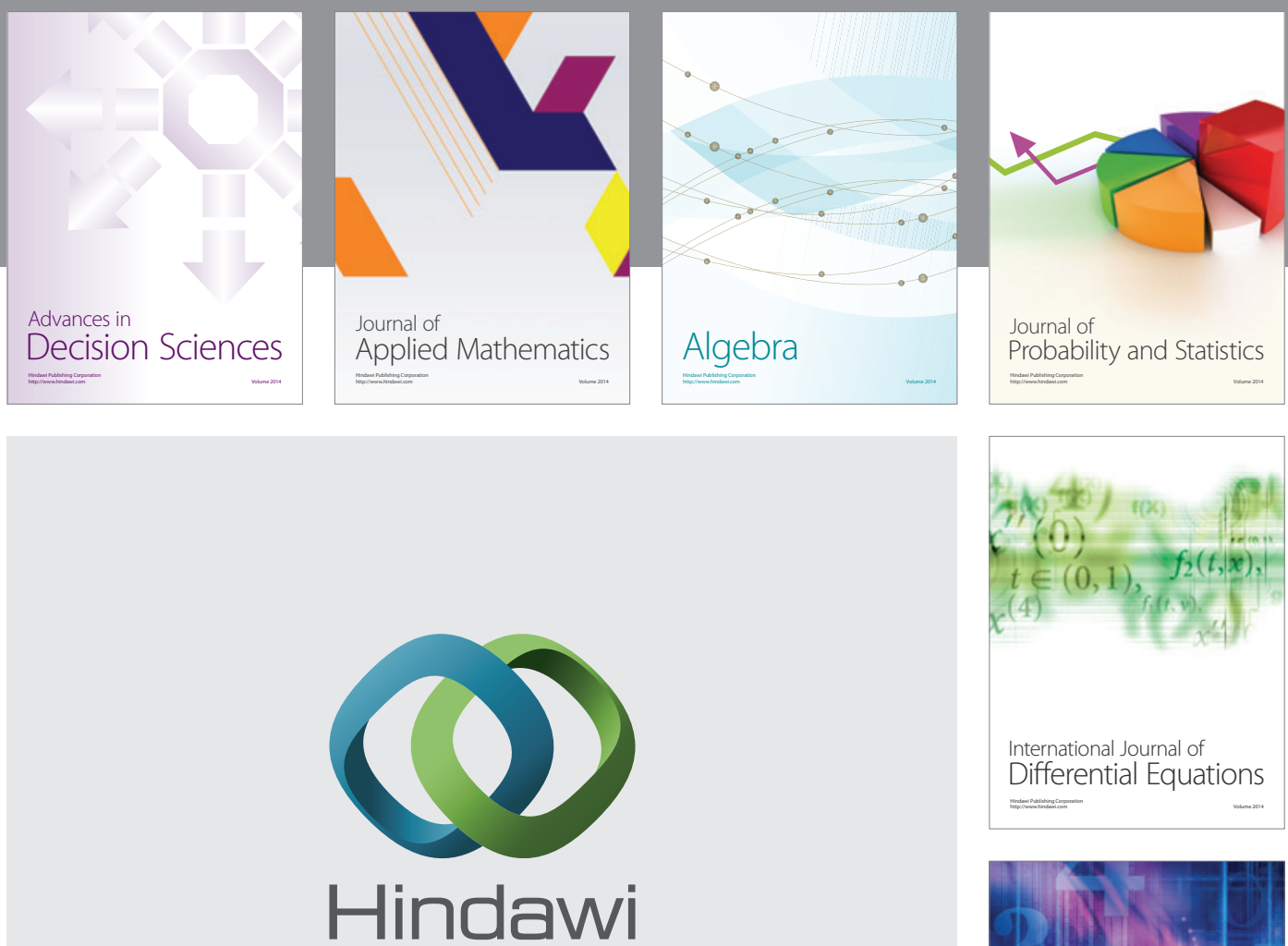

Submit your manuscripts at http://www.hindawi.com
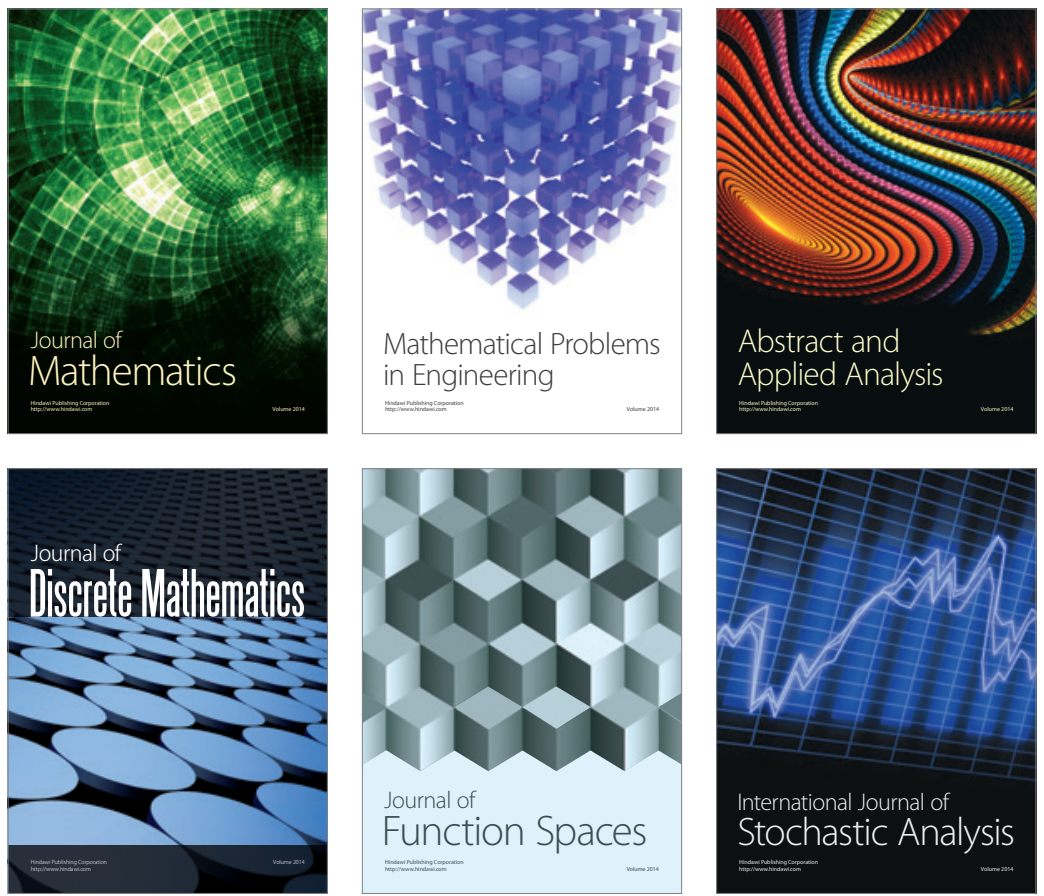

Journal of

Function Spaces

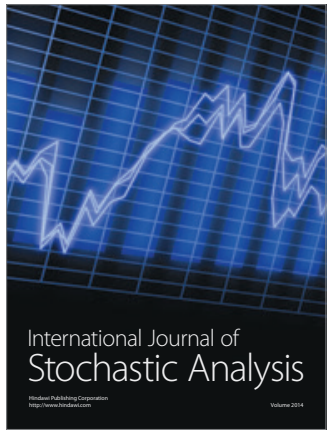

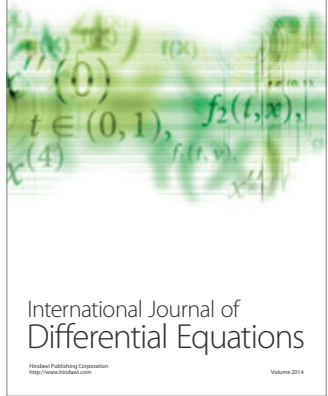
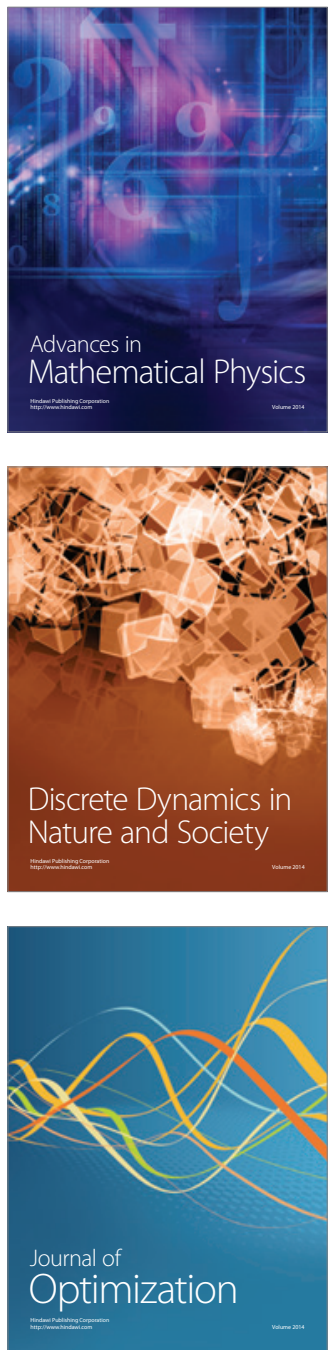